\title{
An algorithm for fast determination of complex moduli
}

\author{
Grethe Winther and Ole Kramer \\ Department of Chemistry, University of Copenhagen, \\ Universitetsparken 5, DK-2100 Copenhagen, Denmark \\ Jeppe C. Dyre \\ Institute of Mathematics and Physics (IMFUFA), Roskilde University, \\ P.O. Box 260, DK-4000 Roskilde, Denmark
}

(Received 26 May 1993; accepted 26 February 1994)

\begin{abstract}
Synopsis
Traditionally, the complex modulus is determined by Fourier analysis of steady statc oscillatory data. However, steady state is not obtained immediately and data from the first period of oscillation must therefore be discarded. In the present work a recursive analysis algorithm for the determination of complex moduli from oscillatory data of the first period, which includes a transient response alongside the steady state response, is derived. The algorithm is based on Boltzmann's principle of supcrposition. At any given timc, the analysis algorithm provides the best possible estimate of the complex modulus on the basis of the information available at that time, i.e., the stress and strain history. The analysis algorithm has been tested on simulated data from a mathematical model of an amorphous polymer. The tests show that the new analysis algorithm can determine the dynamic mechanical properties with very good accuracy from oscillatory data of the first period, where Fourier analysis fails. Thus, use of the new algorithm allows a reduction of the experiment time by a factor of 2 for experiments with a sufficiently high signal-to-noise ratio to avoid averaging over several periods. This reduction of the measurement time is particularly important for experiments at very low frequencies where the long measurement times seriously limit the number of runs per day.
\end{abstract}

\section{INTRODUCTION}

Computers have found extensive use in rheology as analytical tools, as well as for control and data acquisition in rheological instrumentation. Computers can generate arbitrary stress or strain inputs and can be used for any kind of mathematical analysis of the data. This possibility raises the question of how to obtain maximum information about a material in a given experiment time.

In the linear regime, a material is characterized in the frequency domain by its complex modulus function. To measure the complex modulus, a sinusoidal stress or strain is typically applied to the sample and the strain or stress response is recorded. Steady state data are then Fourier analyzed to calculate the complex modulus at the input frequency. This procedure is repeated for other frequencies until the desired frequency range is covered.

Holly et al. (1988) showed how to shorten the experiment time by choosing the input as a sum of sinusoids of different frequencies, a method which has been implemented on 
commercial instruments by, e.g., Rheometrics and Bohlin. However, one still has to wait for steady state before analysis by Fourier decomposition gives the correct complex modulus.

Fourier decomposition is a general method for data analysis in the frequency domain. The method is based on the knowledge that, in the linear regime, a sinusoidal input results in a sinusoidal response at steady state. For viscoelastic materials, however, steady state is not obtained immediately. A transient response is present alongside the steady state response and Fourier analysis does not give the correct complex modulus until this transient response has died off.

It has been shown [Winther (1993)] that the error introduced by performing Fourier analysis on data from the first period of a single frequency can be as large as $16 \%$. Discarding all data taken during the first period and only analyzing data from the second period gives the correct modulus within $1 \%$. When more frequencies are applied simultaneously, the errors increase because the analyzed data include transient responses from all applied frequencies.

An algorithm for determination of the complex modulus of linear viscoelastic materials from data taken before steady state [Winther et al. (1993)] is derived and tested in detail in the present paper. The analysis algorithm is based on Boltzmann's principle of superposition. A least-squares fit to a discrete "relaxation time spectrum" is obtained and the complex modulus may then be calculated from the obtained weights. The term relaxation time spectrum is written in quotation marks because it merely is used as a parametrization. Negative weights are allowed since the aim only is to determine the complex modulus. The algorithm employs recursive linear equations and utilizes well-known principles from time-series analysis, dating back to Gauss [Young (1984)].

The algorithm is especially useful for experiments at low frequencies where the absolute measurement time, and therefore also the time saved, is long. If the principle of time-temperature superposition does not apply to the sample, as for example in the case of biological systems such as gelatin and fibrin networks, it may be desirable to make measurements at frequencies of less than $10^{-4} \mathrm{rad} \mathrm{s}^{-1}$, corresponding to periods of the order of a day. For such experiments it is particularly unfortunate that data collected during the first period must be discarded, because steady state has to be obtained before Fourier analysis gives the correct result.

Many data points can usually be taken during the first period so it is unnecessary to average over several periods to suppress the influence of noise. Inertial effects are furthermore negligible at low frequencies so that perfect sinusoidal strains can be realized.

Experimental data covering all the characteristic regions of viscoelastic behavior are not easily obtained, partly because 10-12 decades of dynamic mechanical moduli must be covered, and partly because we are particularly interested in the initial part of the data which usually are discarded by computerized rheometers. However, a full survey of the capabilities and limitations of the algorithm can be performed by computer simulations of "experiments" on a mathematical model which exhibits all the characteristics of a polymer, i.e., glassy, glass transition, plateau, terminal and liquid regions, and in a fraction of the time required for actual experiments.

This paper presents the results of analyzing the simulated stress response from such a mathematical model hoth hy Fourier decomposition and by the new analysis algorithm in order to compare their abilities to obtain the correct complex modulus function. The simulations demonstrate that the complex modulus can be determined from data of the first period which include a significant transient response. Thus, a reduction of the experiment time by a factor of 2 is accomplished with the new algorithm, since the tradi- 
tional Fourier analysis uses data from the second period, discarding the transient data from the first period.

Application of the algorithm to more limited experimental data from two different rheometers is planned.

\section{THE NEW ANALYSIS ALGORITHM}

This section addresses the problem of obtaining the best possible estimate of the complex modulus function from arbitrary data, i.e., data that do not necessarily come from an experiment where the input is periodic. In this paper the input is taken to be the strain but analogous equations may be derived for stress inputs. If $\gamma$ denotes the shear applied to a viscoelastic liquid and $\sigma$ the shear stress, Boltzmann's principle of superposition gives the general linear relationship between them as described, e.g., by Bird et al. (1987):

$$
\sigma(t)=-\int_{0}^{\infty} G\left(t^{\prime}\right) \frac{d \chi\left(t-t^{\prime}\right)}{d t^{\prime}} d t^{\prime}
$$

Here, $G\left(t^{\prime}\right)$ is the relaxation modulus, i.e., the stress at time $t^{\prime}$ after a unit step strain is applied. The relaxation modulus is expressed as a function of exponential decays [see, e.g. Ferry (1980)]:

$$
G\left(t^{\prime}\right)=\int_{-\infty}^{\infty} H(\tau) e^{-t^{\prime} / \tau} d \ln \tau
$$

where $\tau$ is the relaxation time and $H(\tau)$ is the relaxation time spectrum.

It has been shown that just a few relaxation times per decade are sufficient to describe the relaxation time spectrum [Baumgaertel and Winter (1992)] and Eq. (2) can be rewritten as

$$
G\left(t^{\prime}\right)=\sum_{j=1}^{n} G_{j} e^{-t^{\prime} / \tau_{j}}
$$

The $G_{j}$ 's of Eq. (3) correspond to a discretization of the function $H(\tau)$ appearing in $\mathrm{Eq}$. (2). The relaxation times are usually chosen to be uniformly distributed on a logarithmic scale. The new analysis algorithm is based on Eqs. (1)-(3) and determines a number of coefficients which for convenience also are called $G_{j}$ although they do not constitute a true relaxation time spectrum. The reasons for this will become clear later.

In many experiments data acquisition is performed by a digital computer, and stress and strain are both known only at discrete times with a spacing $\Delta t$. Discretizing the integral in Eq. (1) gives

$$
\begin{aligned}
\sigma(k \Delta t) & \left.\left.=G\left(\frac{1}{2} \Delta t\right)\{\gamma(k \Delta t)-\gamma(k-1) \Delta t]\right\}+G\left(\frac{3}{2} \Delta t\right)\{\gamma(k-1) \Delta t]-\gamma[(k-2) \Delta t]\right\}+\ldots \\
& \left.=G\left(\frac{1}{2} \Delta t\right) \gamma(k \Delta t)-\sum_{m=1}^{\infty}\left\{G\left[\left(m-\frac{1}{2}\right) \Delta t\right]-G\left[\left(m+\frac{1}{2}\right) \Delta t\right]\right\} \gamma(k-m) \Delta t\right] .
\end{aligned}
$$

Substituting Eq. (3) into Eq. (4) yields the predicted stress for given coefficients, $G_{j}$. Below, $\sigma_{\mathrm{pr}}$ denotes the predicted stress to distinguish it from the actually measured stress, $\sigma$. Equation (4) is then rewritten as 


$$
\sigma_{\mathrm{pr}}(k \Delta t)=\sum_{j=1}^{n} G_{j} f(j, k)
$$

where

$$
\begin{aligned}
f(j, k) & =e^{-\Delta t /\left(2 \tau_{j}\right)} \gamma(k \Delta t)-\left[e^{\Delta t /\left(2 \tau_{j}\right)}-e^{-\Delta t /\left(2 \tau_{j}\right)}\right] \sum_{m=1}^{\infty} e^{-m \Delta t / \tau_{j}} \gamma[(k-m) \Delta t] \\
& =e^{-\Delta t /\left(2 \tau_{j}\right)} \gamma(k \Delta t)-2 \sinh \left(\frac{\Delta t}{2 \tau_{j}}\right) \sum_{m=1}^{\infty} e^{-m \Delta t / \tau_{j}} \gamma[(k-m) \Delta t] .
\end{aligned}
$$

Some loss of information occurs when the continuous function is discretized. Relaxation times that are much smaller than the sampling interval, $\Delta t$, cannot be properly incorporated into the discrete model because their contribution to the total relaxation of the material occurs between two data points. The inability to handle small relaxation times can be seen mathematically in $\mathrm{Eq}$. (6) where the factor $f(j, k)$, which describes the stress contribution from the relaxation time, $\tau_{j}$, quickly vanishes as $\Delta t / \tau_{j} \rightarrow \infty$.

We now turn to the problem of estimating the coefficients, $G_{j}$, from given experimental data, the relaxation times, $\tau_{j}$, being fixed in this study. The $G_{j}$ 's are determined so that the predicted stress matches the measured stress as clnsely as possible. To make maximum use of the data, the entire measured stress history up to a given time should be approximated as well as possible by the predicted stress. The simplest approach to this problem is to optimize in the least-squares sense since this leads to linear equations for the coefficients [Young 1984]. Adopting this strategy, the $G_{j}$ 's at any time $t=k \Delta t$ are to be determined from

$$
\frac{\partial}{\partial G_{i}} \sum_{p=0}^{k}\left[\sigma_{\mathrm{pr}}(p \Delta t)-\sigma(p \Delta t)\right]^{2}=0,
$$

or

$$
\sum_{p-0}^{k}\left[\sigma_{\mathrm{pr}}(p \Delta t)-\sigma(p \Delta t)\right] 2 f(i, p)=0
$$

It is assumed that the errors can be considered constant as is the case for white noise.

Introducing the quantities

$$
\begin{aligned}
& A_{i j}(k)=\sum_{p=0}^{k} f(i, p) f(j, p), \\
& b_{i}(k)=\sum_{p=0}^{k} f(i, p) \sigma(p \Delta t),
\end{aligned}
$$

and remembering Eq. (5), Eq. (8) becomes

$$
\sum_{j=1}^{n} A_{i j}(k) G_{j}=b_{i}(k) .
$$


Thus, at any given time, $t=k \Delta t$, the optimum $G_{j}$ 's are determined as the solution of a linear system of equations with a symmetric coefficient matrix. This system of linear equations is ill-posed [Friedrich and Hoffmann (1983); Honerkamp (1989)], i.e., the solution is very sensitive to small fluctuations of the data. However, the determined $G_{j}$ 's, some of which may be negative, fit the data, and as demonstrated below give accurate complex moduli even though they do not represent the true relaxation time spectrum. (The relaxation time spectrum may subsequently by derived from the determined complex modulus by one of the recently published methods [Baumgaertel and Winter (1989); Carrot et al. (1992); Elster et al. (1991); Elster and Honerkamp (1991); Emri and Tschoegl (1993); Honerkamp (1989); Honerkamp and Weese (1993)].)

The system of equations is well suited to a recursive treatment, since the equations at time $t=(k+1) \Delta t$ can be found from the equations one time step earlier:

$$
\begin{aligned}
& A_{i j}(k+1)=A_{i j}(k)+f(i, k+1) f(j, k+1) \\
& \left.b_{i}(k+1)=b_{i}(k)+f(i, k+1) \sigma(k+1) \Delta t\right] .
\end{aligned}
$$

What makes the recursive approach useful is the fact that the functions $f(j, k)$ from Eq. (6) can also be evaluated recursively:

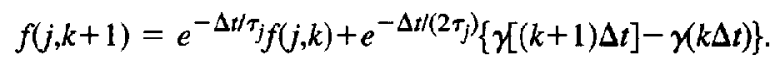

The recursive formulation means that it is not necessary to store all strain and stress data during the experiment to perform a least-squares fit to a fixed set of relaxation times. The matrix $A_{i j}(k)$ and the right-hand side $b_{i}(k)$ of Eq. (10) carry all information needed for determination of the optimum $G_{j}$ 's at any given time--even for an experiment of long duration.

\section{Complex modulus}

Application of a sinusoidal strain gives a sinusoidal stress in the steady state. In the case of continuous strain and stress data, the complex modulus is found by substituting Eq. (3) and a sinusoidal strain into Eq. (1) for $t \rightarrow \infty$ which gives the simple and wellknown formula

$$
G^{*}(\omega)=\sum_{j=1}^{n} G_{j} \frac{i \omega \tau_{j}}{1+i \omega \tau_{j}}
$$

The new algorithm is applied to discrete stress and strain data, and the complex modulus is found by substitution of a sinusoidal strain into Eq. (5). The result is

$$
G^{*}(\omega)=\sum_{j=1}^{n} G_{j} e^{-\Delta t /\left(2 \tau_{j}\right)} \frac{1-e^{-i \omega \Delta t}}{1-e^{-\Delta t \tau_{j}} e^{-i \omega \Delta t}},
$$

which is derived in Appendix A. In the limit $\Delta t \rightarrow 0$, Eq. (14) reduces to the continuous case in Eq. (13).

Again some information is lost by discretization. The problem of aliasing frequencies arises so that components of frequencies that differ by the sampling frequency cannot be distinguished. The discrete expression for $G^{*}(\omega)$ is thus periodic with a period of $2 \pi / \Delta t$.

\section{COMPUTER SIMULATIONS}

The new analysis algorithm can handle any strain history-periodic or aperiodic-but a sum of a fundamental sine wave and seven harmonics is chosen in the present paper. 


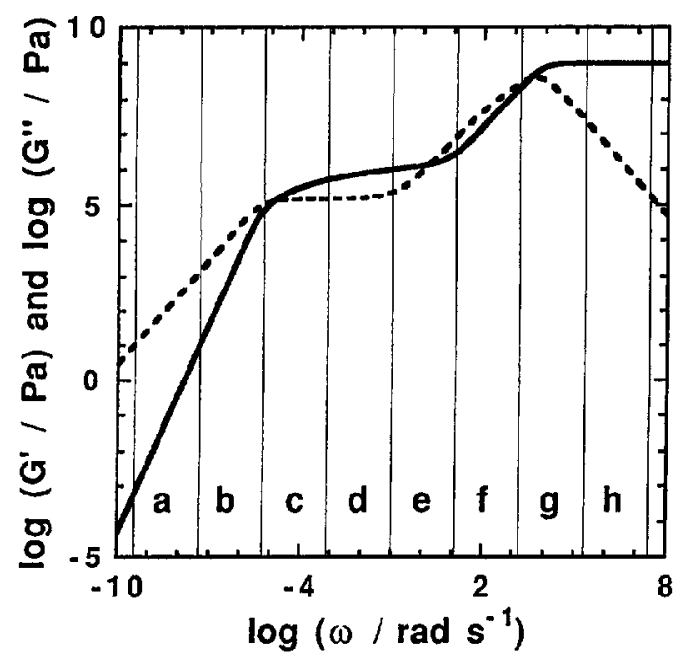

FIG. 1. Storage modulus, $G^{\prime}(-)$, and loss modulus, $G^{\prime \prime}(--)$, of the polymer model given by Eq. (18) which is derived from the relaxation time spectrum given by Eq. (15).

The sum of the eight strain frequencies is applied to a mathematical polymer model described below and the resulting stress response is analyzed. The spacing between the frequencies on a logarithmic scale is $\log 2$ and each simulated experiment thus covers a factor of 128 in frequency, i.e., a little more than two decades. After each simulated experiment, the $G_{j}$ 's are calculated from Eq. (10) and used to calculate the complex modulus from the discrete expression in Eq. (14).

\section{The mathematical polymer model}

The mathematical model used in this study is an approximation of an amorphous polymer. Its relaxation time spectrum is a box and wedge distribution in a double logarithmic plot [Aklonis and MacKnight (1983)] and given by

$$
\begin{aligned}
& H(\tau)=\frac{A}{\tau} ; \quad \tau_{1}<\tau<\tau_{2} \\
& H(\tau)=B ; \quad \tau_{2}<\tau<\tau_{3} .
\end{aligned}
$$

The storage and loss moduli of the polymer model are shown in Fig. 1 with $\tau_{1}=10^{-4} \mathrm{~s}$, $\tau_{2}=1 \mathrm{~s}, \tau_{3}=3 \times 10^{5} \mathrm{~s}, A=10^{5} \mathrm{~Pa} \mathrm{~s}$, and $B=10^{5} \mathrm{~Pa}$. These values are used in all simulations. Eight different sections are indicated in Fig. 1, covering all the possible mechanical properties of an amorphous polymer. The width of each section is chosen so that application of the eight frequencies, covering a factor of 128 , gives an overlap of one frequency between the different sections.

Please note that the slope of the wedge is considerably larger than the slope usually found for actual polymers [Ferry (1980)]. This means that short relaxation times contribute considerably to the complex modulus. As discussed later in the paper, such contributions may be problematic for the new algorithm and use of this polymer model is therefore a very critical test of the algorithm. 
In the computer simulations the strain input is applied to the system at equilibrium at $t=0$ and the exact stress response of the polymer model is calculated from Eqs. (1) and (2). For a single sinusoid of amplitude $\gamma_{0}$ started at $t=0$ the result is

$$
\sigma(t)=\sigma_{\mathrm{ss}}(t)+\sigma_{\mathrm{tr}}(t),
$$

where $\sigma_{\mathrm{ss}}(t)$ is the steady state response and $\sigma_{\mathrm{tr}}(t)$ is the transient response.

The steady state stress response $\sigma_{\mathrm{ss}}(t)$ is given by

$$
\sigma_{\mathrm{ss}}(t)=\gamma_{0} G^{\prime}(\omega) \sin (\omega t)+\gamma_{0} G^{\prime \prime}(\omega) \cos (\omega t),
$$

where $G^{\prime}(\omega)$ and $G^{\prime \prime}(\omega)$ are derived for this particular case in Appendix B and given by

$$
\begin{gathered}
G^{\prime}(\omega)=A \omega\left[\arctan \left(\omega \tau_{2}\right)-\arctan \left(\omega \tau_{1}\right)\right]+\frac{B}{2} \ln \left(\frac{1+\left(\omega \tau_{3}\right)^{2}}{1+\left(\omega \tau_{2}\right)^{2}}\right), \\
G^{\prime \prime}(\omega)=A \omega \ln \left(\frac{\tau_{2}}{\tau_{1}}\right)-\frac{A \omega}{2} \ln \left(\frac{1+\left(\omega \tau_{2}\right)^{2}}{1+\left(\omega \tau_{1}\right)^{2}}\right)+B\left[\arctan \left(\omega \tau_{3}\right)-\arctan \left(\omega \tau_{2}\right)\right] .
\end{gathered}
$$

where, $A, B, \tau_{1}, \tau_{2}$, and $\tau_{3}$ are defined in Eq. (15).

The transient stress response $\sigma_{\mathrm{tr}}(t)$ is also derived in Appendix B and given by

$$
\begin{aligned}
\sigma_{\mathrm{tr}}(t)= & \gamma_{0}\left\{A \omega\left[R_{1}(t)-R_{2}(t)\right]+B\left[I_{3}(t)-I_{2}(t)\right]\right\} \cos (\omega t) \\
& +\gamma_{0}\left\{-A \omega\left[I_{1}(t)-I_{2}(t)\right]+B\left[R_{3}(t)-R_{2}(t)\right]\right\} \sin (\omega t)
\end{aligned}
$$

where

$$
R_{m}(t)+i I_{m}(t)=E_{1}\left(\frac{t}{\tau_{m}}+i \omega t\right) \quad(m=1,2,3) .
$$

Here $E_{1}$ denotes the exponential integral as defined by Abramowitz and Stegun (1966) (see Appendix B). Both $R_{j}(t)$ and $I_{j}(t)$ go to zero as $t \rightarrow \infty$, implying that the transient stress response dies off. The numerical implementation is given in Appendix $C$.

The total stress response from an input containing eight frequencies is the sum of the responses originating from each frequency.

At any time, the $G_{j}$ 's may be calculated by solving the linear equations given as Eq. (10). It is of course not necessary to solve the equations for each updating. The equations are solved by the Doolittle method described by Ralston and Rabinowitz (1986).

\section{RESULTS AND DISCUSSION}

A series of simulations were performed in order to establish the parameters which give the most accurate determination of the complex modulus function. The relationship between the applied frequencies, the relaxation times included in the fit and the sampling interval were varied. In the following we summarize the results of this optimization, and we then compare the complex moduli obtained with the new algorithm to those obtained with Fourier analysis.

\section{Optimization of the algorithm}

Not surprisingly, it was found that the relaxation times to be included in the fit depend on the frequency content of the strain input. The contribution from a single relaxation time, $\tau$, to the complex modulus at a given frequency, $\omega$, depends on the value of $\omega \tau$. For small values of $\omega \tau$, the contribution to $G^{\prime}$ is proportional to $\omega^{2}$ and the contribution to $G^{\prime \prime}$ is proportional to $\omega$. For large values of $\omega \tau$, the contribution to $G^{\prime}$ is independent of 
$\omega$ and the contribution to $G^{\prime \prime}$ is proportional to $\omega^{-1}$. The contributions from very small or from very large relaxation times are consequently unresolvable because they cannot be distinguished by their frequency dependence.

Only relaxation times in an interval around $\omega \tau \approx 1$ for each frequency are included in the fit due to the above-mentioned problems with unresolvable relaxation times. Since the eight frequencies used in a simulation only cover about two decades, it means that the coefficients $G_{j}$ obtained from Eq. (10) will not reflect the true relaxation time spectrum when relaxation times outside the included range contribute considerably to the properties of the material. The $G_{j}$ 's simply represent the best fit to the stress and strain data and this means that negative $G_{j}$ 's may occur. However, the complex modulus calculated from these coefficients using Eq. (14) is nevertheless correct.

The properties of the mathematical polymer model chosen for this study contain very large contributions from short relaxation times which cannot always be included and in this respect the simulations constitute a "worst case."

The optimal spacing of the relaxation times turned out to be about three relaxation times per decade. Baumgaertel and Winter (1992) have demonstrated that 1.5 relaxation times per decade are sufficient to discretize a continuous spectrum satisfactorily. If variable relaxation times are used even fewer relaxation times are needed to characterize a material [Baumgaertel and Winter (1989); Carrot et al. (1992)]. However, in the new algorithm the determined coefficients only represent the best fit and cannot be regarded as a discrete relaxation time spectrum. It is therefore not surprising that more coefficients are needed as fitting variables in regions where the unresolvable relaxation times give large contributions.

As already mentioned, the algorithm cannot handle large values of $\Delta t / \tau$. The optimal value of the smallest relaxation time, $\tau_{\min }$, turns out to be the sampling interval, $\Delta t$. The optimal $\tau_{\max }$ is around $10 / \omega_{\min }\left(\omega_{\min }\right.$ given in $\left.\mathrm{rad} / \mathrm{s}\right)$.

The relationship between the values of the applied frequencies and the sampling interval has also been varied. It turns out that it is necessary to sample about 60 data points per period of $\omega_{\max }$. This means that approximately $8000(\approx 60 \times 128)$ data points will be sampled during a period of $\omega_{\min }=\omega_{\max } / 128$.

The reader must bear in mind that the established optimal relationships between frequencies, relaxation times, and the sampling interval may not hold if the number of frequencies or the frequency interval of the applied strain are changed. A new set of optimal parameters will then have to be found.

The algorithm is flexible with respect to the duration of the experiment. At any time, Eq. (10) can be solved for the best estimate of the coefficients $G_{j}$ and thereby the complex modulus. However, the lowest frequency for which the calculated complex modulus is valid is determined by the duration of the experiment since the data must obviously contain information about the desired time scale. If the complex modulus is calculated after an experiment time, $T$, less than a full period of $\omega_{\min }$, the calculated modulus cannot be expected to be valid down to $\omega_{\min }$ but only down to about $\omega=(2 \pi) /$ $T$. On the other hand, continuation of the experiment for more than a full period of $\omega_{\min }$ does not improve the results because steady state will be obtained so that the data do not contain new information. In this paper, however, we only present results from simulations covering one full period of the lowest frequency.

For convenience, relaxation times which are powers of two, i.e., 3.3 relaxation times per decade, are used in the rest of this paper. The frequencies are also chosen so that the number of data points per period is a power of two, i.e., $\omega_{\max }=(2 \pi) /(64 \Delta t) \mathrm{rad} / \mathrm{s}$ and $\omega_{\min }=(2 \pi) /(128 \times 64 \Delta t) \mathrm{rad} / \mathrm{s}$. Consequently, the number of data points in one period 
of the lowest frequency is 8192 . The fitting parameters are the $15 G_{j}$ 's of Eq. (10), corresponding to the relaxation times $\tau_{i}=2^{i} \Delta t, i=0,1, \ldots, 14$.

For each data point, the values of the quantities $f(j, k), A_{i j}(k)$, and $h_{i}(k)$ [Eqs. (11) and (12)] are updated. On a $50 \mathrm{MHz} 486 \mathrm{DX} 2 \mathrm{PC}$ each update took about $10 \mathrm{~ms}$, allowing a maximum sampling frequency of about $100 \mathrm{~Hz}$ in an experimental situation. This corresponds to a maximum excitation frequency of about $1.6 \mathrm{~Hz}(=10 \mathrm{rad} / \mathrm{s})$ because the sampling frequency must be about 60 times higher than the highest excitation frequency. It shows that modern computers are fast enough to implement the algorithm recursively.

\section{Determination of complex moduli}

Using the oplinized parameters developed in the previous section, the complex moduli of the mathematical polymer model are calculated with the new algorithm and compared to the results obtained with traditional Fourier decomposition. Only data covering the first period of the lowest applied frequency are analyzed which means that the results from the Fourier analysis may be invalid due to the transient stress component.

In order to test both the fundamental properties of the algorithm and its usefulness in a realistic experimental situation, the simulations have been carried out without noise as well as with white noise added to the stress. The strain amplitudes for the eight applied frequencies have been adjusted to give equal stress amplitudes before each simulation. In this way all frequencics are affected equally by the noise present in some of the simulations.

In Fig. 2 the relative errors of $G^{\prime}$ and $G^{\prime \prime}$ obtained from noise-free data covering the first period of the lowest applied frequency are shown for both Fourier analysis and the new algorithm. The letters denoting the subfigures refer to the sections indicated in Fig. 1.

In sections $b-g$, the new analysis algorithm determines the complex modulus accurately in the applied frequency range. In several of these regions, the errors are very small even for about a third of a decade on either side of the frequency range applied. The moduli obtained by Fourier decomposition are too low in sections $\mathrm{c}-\mathrm{g}$ because the analyzed data include a significant transient response and Fourier analysis is not valid. These simulations show that the complex modulus can be determined from initial data which include a transient response, simply by substituting Fourier analysis with the new analysis algorithm.

In the liquid and glassy zones (Fig. 2a and h), the relaxation times of the mathematical polymer model are either much smaller or much larger than the time scale probed by the applied frequencies. The transient response is therefore very small and Fourier decomposition gives good results. The storage and loss moduli differ by several orders of magnitude in these regions and the new analysis algorithm is only capable of determining the larger part well. In the liquid zone the storage modulus fluctuates wildly between errors of several thousand percent so it is easy to detect that the result is incorrect. In the glassy zone the loss modulus fluctuates somewhat in the range of applied frequencies. However, the inability to determine the smaller part in these zones is not a serious problem since materials are well characterized by just a single number, either the viscosity or the glass modulus.

Figure 3 again shows the results from the first period but now Gaussian white noise has been added to the stress. The dispersion of the noise is $2 \%$ of the maximum stress value. In sections $\mathrm{c}-\mathrm{g}$, the largest error of the moduli found by the new analysis algorithm is about $5 \%$ and occurs for the lowest applied frequency. The errors for the higher frequencies are smaller except for section $\mathrm{g}$, where the loss modulus becomes small at the 

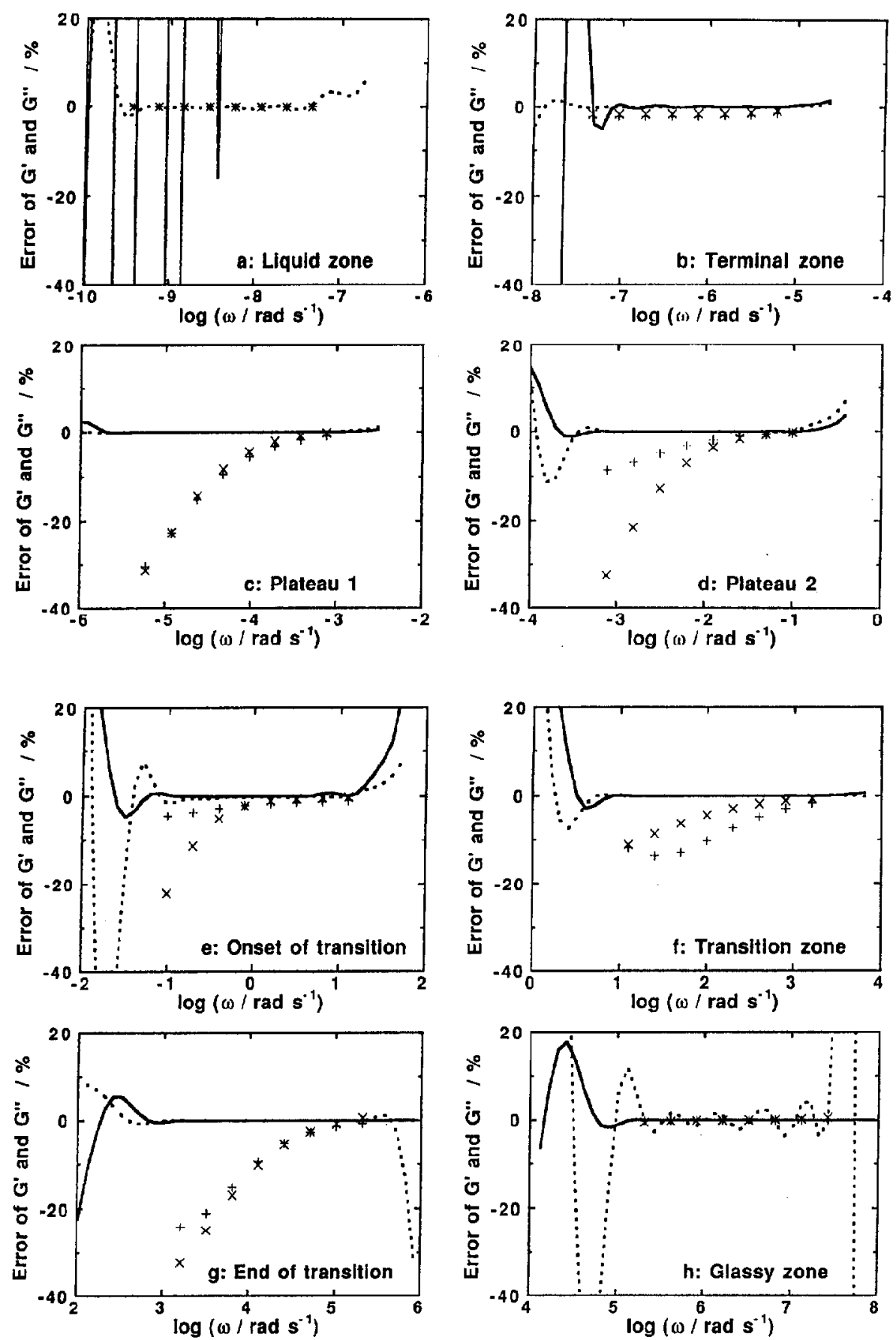

FIG. 2. Results obtained from noise-free data from the first period of the lowest frequency. Errors of $G^{\prime}$ and $G^{\prime \prime}$ (in percent) from Fourier decomposition $(+, X)$ and the new analysis algorithm $(-,--)$ are taken relative to the true moduli shown in Fig. 1. The figure shows that the new analysis algorithm gives accurate moduli where Fourier analysis fails due to the transient response. 

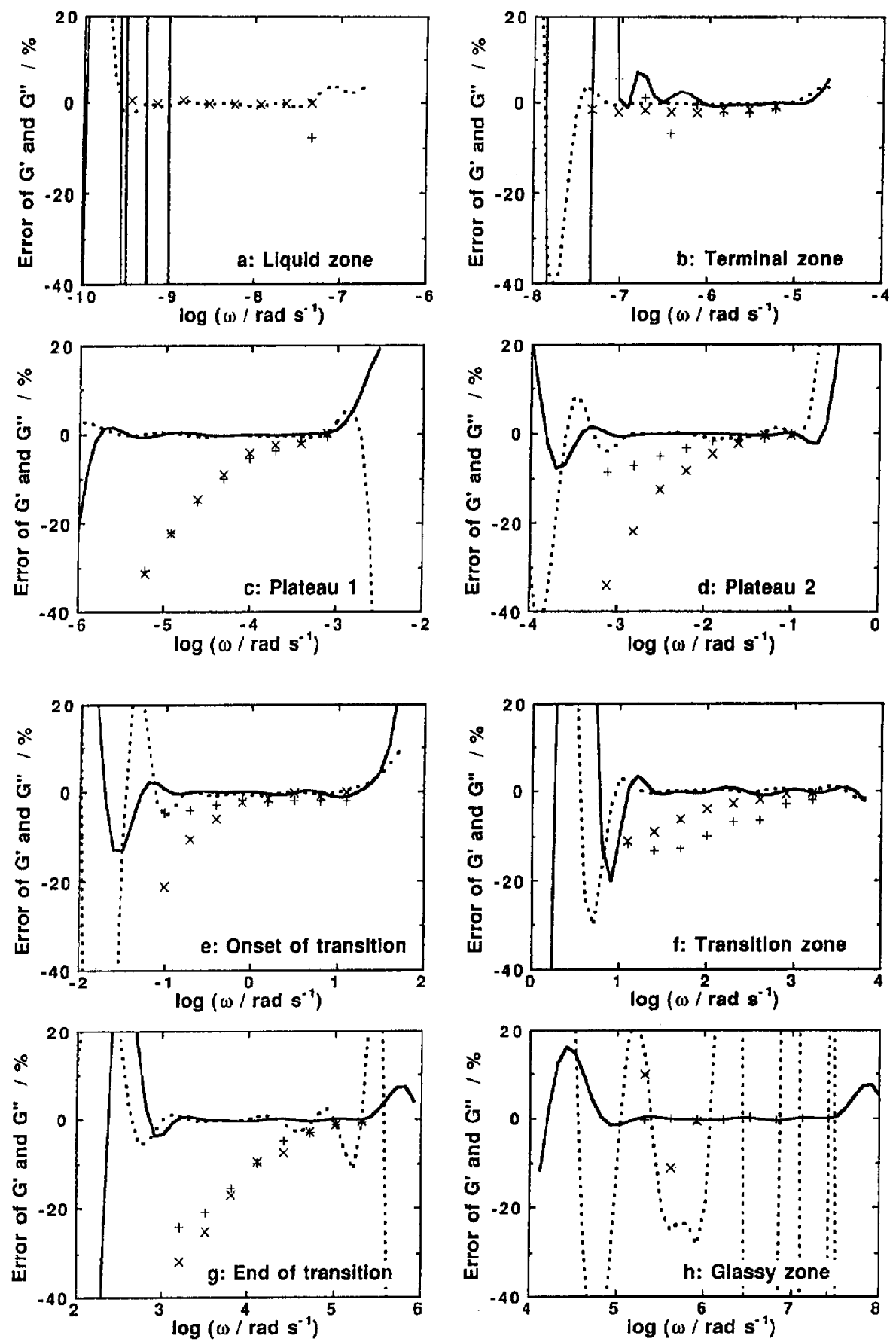

FIG. 3. Noisy data from the first period of the lowest applied frequency are analyzed by both Fourier decomposition and the new analysis algorithm. Gaussian white noise with a dispersion of $2 \%$ of the maximum stress value has been added to the stress. The errors of $G^{\prime}$ and $G^{\prime \prime}$ obtained by Fourier decomposition $(+, X)$ and the new analysis algorithm (-,--) are taken relative to the true moduli shown in Fig. 1. By comparison to Fig. 2 it can be seen that both Fourier analysis and the new analysis algorithm give poorer results when noise is present. 
highest frequencies (see Fig. 1). The new analysis algorithm generally determines the moduli within a few percent for frequencies which are about a decade lower than the frequencies where Fourier analysis gives the correct moduli. However, the moduli cannot be determined at frequencies which are lower than the applied frequencies when noise is present. The results from Fourier decomposition are erroneous both because of the transients, as in Fig. 2, and the noise.

In contrast to the noise-free simulations in Fig. 2, Fourier analysis cannot determine the smaller part of the complex modulus in the liquid and glassy zones (Fig. 3a, b, and $\mathbf{h}$ ). For both Fourier analysis and the new analysis algorithm, the errors are too large to be plotted on the scale used in Fig. 3. However, Fourier analysis will eventually succeed if data from a sufficient number of periods are averaged.

\section{CONCLUSION}

A thorough survey of the optimal conditions and potential capabilities of a new algorithm for analyzing nonsteady state oscillatory data has been performed, using computer simulations.

The results demonstrate that the new analysis algorithm makes it possible to obtain the complex modulus from oscillatory data taken before steady state is obtained, i.e., during the first period. This means that the experiment time will be reduced by a factor of 2 , since traditional Fourier analysis requires steady state data which are obtained in the second period.

The computer simulations show that the new analysis algorithm is successful even on oscillatory data with white noise added. The next step is application of the algorithm to true, but more limited, experimental data.

\section{ACKNOWLEDGMENTS}

We wish to thank Jakob Stoustrup from the Danish Technical University for helpful discussions and Professor N. W. Tschoegl for constructive criticism of an early version of the manuscript. Financial support from the Danish Natural Science Research Council through Grants No. 11-9586 and 11-0425 is gratefully acknowledged.

\section{APPENDIX A: DERIVATION OF THE DISCRETE EXPRESSION FOR THE COMPLEX MODULUS}

Application of a sinusoidal strain gives a sinusoidal stress in the steady state:

$$
\sigma(t)=\sigma_{0}^{*} e^{i \omega t}, \quad \gamma(t)=\gamma_{0}^{*} e^{i \omega t}
$$

where $\sigma_{0}^{*}$ and $\gamma_{0}^{*}$ are the complex amplitudes. Substitution of this into Eq. (5) gives

$$
\sigma_{0}^{*} e^{i \omega k \Delta t}=\sum_{j=1}^{n} G_{j} f_{\omega}^{*}(j, k)
$$

where $f_{\omega}^{*}(j, k)$ is given by

$$
\begin{aligned}
f_{\omega}^{*}(j, k) & =\gamma_{0}^{*} e^{i \omega k \Delta t}\left(e^{-\Delta t /\left(2 \tau_{j}\right)}-2 \sinh \left(\frac{\Delta t}{2 \tau_{j}}\right) \sum_{m=1}^{\infty} e^{-m\left(\Delta t / \tau_{j}+i \omega \Delta t\right)}\right) \\
& =\gamma_{0}^{*} e^{i \omega k \Delta t} e^{-\Delta t /\left(2 \tau_{j}\right)} \frac{1-e^{-i \omega \Delta t}}{1-e^{-\Delta t / \tau_{j}} e^{-i \omega \Delta t}}
\end{aligned}
$$


The last equality is obtained by means of the geometric series:

$$
\sum_{m=1}^{\infty} e^{-(\Delta t / \tau+i \omega \Delta t) m}=\frac{1}{1-e^{-(\Delta t / \tau+i \omega \Delta t)}-1}
$$

For $G^{*}(\omega)$ one thus finds

$$
G^{*}(\omega)=\sum_{j=1}^{n} G_{j} e^{-\Delta t /\left(2 \tau_{j}\right)} \frac{1-e^{-i \omega \Delta t}}{1-e^{-\Delta t / \tau_{j}} e^{-i \omega \Delta t}}
$$

\section{APPENDIX B: EVALUATION OF STRESS RESPONSE FROM THE POLYMER MODEL}

We consider the polymer model defined by Eq. (15). Substituting Eq. (15) into Eq. (2) one finds that the relaxation modulus is given by

$$
G\left(t^{\prime}\right)=A \int_{\tau_{1}}^{\tau_{2}} \frac{e^{-t^{\prime} / \tau}}{\tau} d \ln \tau+B \int_{\tau_{2}}^{\tau_{3}} e^{-t^{\prime} / \tau} d \ln \tau
$$

Introducing the variable $\alpha=1 / \tau$ we find with the obvious definition of the new integration limits

$G\left(t^{\prime}\right)=A \int_{\alpha_{2}}^{\alpha_{1}} e^{-\alpha t^{\prime}} d \alpha+B \int_{\alpha_{3}}^{\alpha_{2}} e^{-\alpha t^{\prime}} \frac{d \alpha}{\alpha}=\frac{A}{t^{\prime}}\left(e^{-\alpha_{2} t^{\prime}}-e^{-\alpha_{1} t^{\prime}}\right)+B \int_{\alpha_{3}}^{\alpha_{2}} e^{-\alpha t^{\prime}} \frac{d \alpha}{\alpha}$

To calculate the stress response from a sinusoidal strain applied at $t=0$ we first note that the strain may be written

$$
\chi(t)=\sin (\omega t)=\operatorname{Im}\left(e^{i \omega t}\right) .
$$

When substituted into Eq. (1) we find

$$
\sigma(t)=\operatorname{Im}\left[e^{i \omega t}(i \omega) \int_{0}^{t} G\left(t^{\prime}\right) e^{-i \omega t^{\prime}} d t^{\prime}\right]
$$

The term of the integral proportional to $A$ is evaluated by introducing a small $\epsilon$ that eventually is to go to zero; this term may be expressed in terms of the exponential integral defined by Abramowitz and Stegun (1966):

$$
E_{1}(z)=\int_{z}^{\infty} e^{-u} \frac{d u}{u}
$$

as follows

$$
\begin{aligned}
\int_{\epsilon}^{t}\left(e^{-\alpha_{2} t^{\prime}}-e^{-\alpha_{1} t^{\prime}}\right) e^{-i \omega t^{\prime}} \frac{d t^{\prime}}{t^{\prime}}= & \int_{\left(\alpha_{2}+i \omega\right) \epsilon}^{\left(\alpha_{2}+i \omega\right) t} e^{-u} \frac{d u}{u}-\int_{\left(\alpha_{1}+i \omega\right) \epsilon}^{\left(\alpha_{1}+i \omega\right) t} e^{-u} \frac{d u}{u} \\
= & E_{1}\left[\left(\alpha_{2}+i \omega\right) \epsilon\right]-E_{1}\left[\left(\alpha_{2}+i \omega\right) t\right] \\
& -E_{1}\left[\left(\alpha_{1}+i \omega\right) \epsilon\right]+E_{1}\left\lfloor\left(\alpha_{1}+i \omega\right) t\right] .
\end{aligned}
$$


We now let $\epsilon$ go to zero by utilizing the asymptotic behavior $E_{1}(z)=-0.577-\ln (z)$ as $z \rightarrow 0$ and thus the term proportional to $A$ reduces to

$$
\ln \left(\frac{\alpha_{1}+i \omega}{\alpha_{2}+i \omega}\right)+E_{1}\left[\left(\alpha_{1}+i \omega\right) t\right]-E_{1}\left[\left(\alpha_{2}+i \omega\right) t\right]
$$

The term proportional to $B$ is the following

$$
\int_{\alpha_{3}}^{\alpha_{2}} \int_{0}^{t} e^{-(\alpha+i \omega) t^{\prime}} d t^{\prime} \frac{d \alpha}{\alpha}=\int_{\alpha_{3}}^{\alpha_{2}}\left(1-e^{-(\alpha+i \omega) t}\right) \frac{1}{\alpha(\alpha+i \omega)} d \alpha
$$

The integral is evaluated by making use of the identity

$$
\frac{1}{\alpha(\alpha+i \omega)}=\frac{1}{i \omega}\left(\frac{1}{\alpha}-\frac{1}{\alpha+i \omega}\right) \text {. }
$$

Thus we find

$$
\int_{\alpha_{3}}^{\alpha_{2}} \frac{1}{\alpha(\alpha+i \omega)} d \alpha=\frac{1}{i \omega}\left[\ln \left(\frac{\alpha_{2}}{\alpha_{3}}\right)-\ln \left(\frac{\alpha_{2}+i \omega}{\alpha_{3}+i \omega}\right)\right]=\frac{1}{i \omega} \ln \left(\frac{1+i \omega / \alpha_{3}}{1+i \omega / \alpha_{2}}\right)
$$

and

$$
\begin{aligned}
-\frac{1}{i \omega} \int_{\alpha_{3}}^{\alpha_{2}} e^{-(\alpha+i \omega) t}\left(\frac{1}{\alpha}-\frac{1}{\alpha+i \omega}\right) d \alpha= & -\frac{1}{i \omega}\left\{\left[E_{1}\left(\alpha_{3} t\right)-E_{1}\left(\alpha_{2} t\right)\right] e^{-i \omega t}\right. \\
& \left.-E_{1}\left[\left(\alpha_{3}+i \omega\right) t\right]+E_{1}\left[\left(\alpha_{2}+i \omega\right) t\right]\right\} .
\end{aligned}
$$

To summarize the calculation, the stress for a sinusoidal strain applied at $t=0$ is given by

$$
\sigma(t)=\operatorname{Im}\left[C(t) e^{i \omega t}\right]
$$

where

$$
\begin{aligned}
C(t)= & A i \omega\left[\ln \left(\frac{\alpha_{1}+i \omega}{\alpha_{2}+i \omega}\right)+E_{1}\left[\left(\alpha_{1}+i \omega\right) t\right]-E_{1}\left[\left(\alpha_{2}+i \omega\right) t\right]\right] \\
& +B\left[\ln \left(\frac{1+i \omega / \alpha_{3}}{1+i \omega / \alpha_{2}}\right)-\left[E_{1}\left(\alpha_{3} t\right)-E_{1}\left(\alpha_{2} t\right)\right] e^{-i \omega t}\right]+B\left\{E_{1}\left[\left(\alpha_{3}+i \omega\right) t\right]-E_{1}\left[\left(\alpha_{2}+i \omega\right) t\right]\right\}
\end{aligned}
$$

It is now straightforward to derive Eqs. (16)-(19).

\section{APPENDIX C: NUMERICAL IMPLEMENTATION}

The exponential integral $E_{1}$ in Eqs. (19)-(20) is approximated by the following expressions ${ }^{1}$ given by Abramowitz and Stegun (1966):

$$
0 \leqslant x \leqslant 1 \text { : }
$$

\footnotetext{
${ }^{1}\left\{a_{0} \ldots a_{5}\right\}=\{-0.57721566, \quad 0.99999193, \quad-0.24991055, \quad 0.05519968, \quad-0.00976004, \quad 0.00107857\}$, $\left\{b_{0}, \ldots b_{3}\right\}=\{0.2677737343, \quad 8.6347608925, \quad 18.0590169730,8.5733287401\}$ and $\left\{c_{0}, \ldots c_{3}\right\}$ $=\{3.9584969228,21.0996530827,25.6329561486,9.5733223454\}$.
} 


$$
\begin{gathered}
E_{1}(x)=a_{0}+a_{1} x+a_{2} x^{2}+a_{3} x^{3}+a_{4} x^{4}+a_{5} x^{5}-\ln (x)+\epsilon_{1}(x) ; \\
\left|\epsilon_{1}(x)\right|<2 \times 10^{-7}
\end{gathered}
$$

and

$$
\begin{gathered}
1<x \leqslant \infty: \\
E_{1}(x)=\frac{e^{-x}}{x}\left(\frac{b_{0}+b_{1} x+b_{2} x^{2}+b_{3} x^{3}+x^{4}}{c_{0}+c_{1} x+c_{2} x^{2}+c_{3} x^{3}+x^{4}}+\epsilon_{2}(x)\right) \\
\left|\epsilon_{2}(x)\right|<2 \times 10^{-8} .
\end{gathered}
$$

The complex variable $t / \tau_{j}+i \omega t$ is used instead of the real value stated in the equations, and Eq. (C1) is used for $\left|t / \tau_{j}+i \omega t\right| \leqslant 1$ and Eq. (C2) for $\left|t / \tau_{j}+i \omega t\right|>1$. In section $\mathrm{h}$ of Fig. $1, t / \tau_{1} \ll \omega t$ during the first period of the lowest frequency and the difference $E_{1}\left(t / \tau_{1}+i \omega t\right)-E_{1}\left(t / \tau_{2}+i \omega t\right)$ is evaluated by the fourth-order Taylor expansion. This expansion is also used to calculate $E_{1}\left(t / \tau_{3}+i \omega t\right)-E_{1}\left(t / \tau_{2}+i \omega t\right)$ for $t / \tau_{2}<\omega t$.

\section{References}

Abramowitz, M. and I. A. Stegun, Handbook of Mathematical Functions (U.S. Department of Commerce, National Bureau of Standards, 1966), Eqs. 5.1.1, 5.1.53, and 5.1.56.

Aklonis, J. J. and W. J. MacKnight, Introduction to Polymer Viscoelasticity, 2nd ed. (Wiley. New York, 1983), pp. $155-156$.

Baumgaertel, M. and H. H. Winter, "Determination of Discrete Relaxation and Retardation Time Spectra from Dynanic Mechanical Data," Rheol. Acta 28, 511-519 (1989).

Baumgaertel, M. and H. H. Winter, "Interrelation between Discrete and Continuous Relaxation Spectra," in Theoretical and Applied Rheology, Proc. XIth Int. Congr. on Rheology, edited by P. Moldenaers and R. Keunings, Brussels, Belgium, 1992, pp. 893-895.

Bird, R. B., R. C. Armstrong, and O. Hassager, Dynamics of Polymeric Liquids, 2nd ed. (Wiley, New York, 1987).

Carrot, C., J. Guillet, J. May, and J. Puaux, "Application of the Marquardt-Levenberg Procedure to the Determination of Discrete Relaxation Spectra," Makromol. Chem., Theory Simul. 1, 215-231 (1992).

Elster, C. and J. Honerkamp, "Modified Maximum Entropy Method and Its Application to Creep Data," Macromolecules 24, 310-314 (1991).

Elster, C., J. Honerkamp, and J. Weese, "Using Regularization Methods for the Determination of Relaxation and Retardation Spectra of Polymeric Liquids," Rheol. Acta 30, 161-174 (1991).

Emri, I. and N. W. Tschoegl, "Generating Line Spectra from Experimental Responses. Part II: Storage and loss functions," Rheol. Acta 32, 322-327 (1993).

Ferry, J. D., Viscoelastic Properties of Polymer Melts, 3rd ed. (Wiley, New York, 1980)

Friedrich, C. and B. Hoffmann, "Nichtkorrekte Aufgaben in der Rheometrie," Rheol. Acta 22, 425-434 (1983).

Holly, E. E., S. K. Venkataraman, F. Chambon, and H. H. Winter, "Fourier Transform Mechanical Spectroscopy of Viscoelastic Materials with Transient Structure," J. Non-Newt. Fluid Mech. 27, 17-26 (1988).

Honerkamp, J., "Ill-posed Problems in Rheology," Rheol. Acta 28, 363-371 (1989).

Honerkamp, J. and J. Weese, "A Nonlinear Regularization Method for the Calculation of Relaxation Spectra," Rheol. Acta 32, 65-73 (1993).

Ralston, A. and P. Rabinowitz, A First Course in Numerical Analysis (McGraw-Hill, Singapore, 1986), pp. 421-430.

Winther, G., Determination of Frequency Dependent Linear Viscoelastic Properties of Polymers, Ph.D. thesis, University of Copenhagen, 1993.

Winther, G., O. Kramer, and J. C. Dyre, "Fast Determination of the Complex Modulus Function," Ann. Trans. Nordic Rheology Society, 1, 49-51 (1993).

Young, P., Recursive Estimation and Time-Series Analysis (Springer, Berlin, 1984). 